\title{
Evaluation of Matrix Effect and Chromatography Efficiency: New Parameters for Validation of Method Development
}

\author{
Eduard Rogatsky and Daniel Stein \\ General Clinical Research Center, Albert Einstein College of Medicine of Yeshiva University, \\ Bronx, New York, USA
}

From the viewpoint of regulatory guidelines, validation of LC-UV and LC-MS based methods have the same requirements. Matrix effects are not considered for most method validations if they do not influence reproducibility or assay linearity. Since matrix effects can strongly suppress ionizaton efficiency and therefore reduce sensitivity, they must be evaluated (and discussed in the context of method development) - prior to method qualification. The severity of matrix effects is directly dependent upon chromatographic performance. We suggest that evaluation of matrix effects and LC efficiency is essential information for method assessment, optimization and transfer to other mass spectrometers, and should be a mandatory part of routine LC/MS method validation. (J Am Soc Mass Spectrom 2005, 16, 1757-1759) @ 2005 American Society for Mass Spectrometry

One of the essential steps in LC/MS method development is the choice of MS or MS/MS mode or the choice between single or triple quadrupole instruments. For routine quantitative analysis (if fragmentation is not essential for identification), a single quadrupole instrument is cost-effective and provides higher analyte signal in SIM mode compared to MRM (SRM), where signal is limited by fragmentation and transmission efficiencies.

LC/MS analysis of low purity biological samples can cause signal suppression, elevated background, and other negative matrix effects. In this situation, it is only possible to increase $\mathrm{S} / \mathrm{N}$ while simultaneously maintaining high throughput performance by implementing the SRM mode. This, of course, implies a much higher cost of initial investment in sophisticated triple quadrupole instruments. An alternative approach to increasing $\mathrm{S} / \mathrm{N}$ is to improve the quality of chromatography purification. This decreases matrix effects and makes possible the use of comparatively less expensive single quadrupole instruments while still achieving high analytical performance. Implementation of assays on single quadrupole mass spectrometers can be a good alternative for non-time intensive or budgeted limited projects. Therefore, enhancement of chromatography by implementation of two-dimensional LC methods or even off-line

Published online September 28, 2005

Address reprint requests to Dr. E. Rogatsky, General Clinical Research Center, Albert Einstein College of Medicine of Yeshiva University, Golding Bldg. Room G02, 1300 Morris Park Ave., Bronx, NY 10461, USA. E-mail: rogatsky@aecom.yu.edu purification of analyte (especially from complex biological matrices) with resultant reduction of matrix effect can significantly improve the sensitivity of mass spectrometers by increasing ionization efficiency of a purer analyte. From the viewpoint of method development and optimization, information about the strength of matrix effects and chromatographic efficiency is important if the goal is to achieve optimum sensitivity.

From a regulatory viewpoint, method validation procedures review the following parameters: precision, recovery, specificity, linearity, limit of detection (LOD) and limit of quantitation (LOQ), ruggedness, and robustness [1]. From the viewpoint of regulatory guidelines, validation of LC-UV and LC-MS based methods have the same requirements. In other words, the mass spectrometer is considered just as another type of passive detector. Matrix effects are not considered for most method validations if they do not influence reproducibility or assay linearity. Not directly addressed are technical issues unique to mass spectrometry based assays such as impact of matrix effects on theoretical maximum sensitivity. As mentioned previously, matrix effects can strongly suppress ionization efficiency and sensitivity, and therefore, must be evaluated (and discussed in the context of method development-before method qualification). Therefore, we would like to suggest that the estimation of the strength of matrix effects is a critical technical aspect of MS based assay development.

Unfortunately, method development is sometimes treated as a technical step and a tool for data generation only. As a result, in papers discussing new methods, the 
discussion may be more focused on the data obtained. Discussion about method development, therefore, is brief and focuses only on validation parameters. From a regulatory viewpoint, there are no qualitatively unacceptable methods if the method is validated and allows reproducible data generation. What is often not appreciated is that even properly validated LC/MS or LC/ MS/MS methods can suffer from loss of sensitivity attributable to matrix effects resulting from insufficient (or inefficient) chromatographic purification.

The gain in sensitivity attributable to chromatographic efficiency might be evaluated as the $\mathrm{S} / \mathrm{N}$ ratio of a real sample injected onto the column (LC/MS) compared to the $\mathrm{S} / \mathrm{N}$ injected directly to source (FIA) [2]. However, FIA provides reliable information only when analyzing relatively pure samples; therefore, this method is generally unacceptable for the majority of complex samples.

We suggest that an initial assessment of the strength of the matrix effect can be made by comparing the average values of the background of an actual complex sample compared to a solvent blank by Q1 SIM. A more precise initial approach is to compare the relative response ( $/ \mathrm{N}$, area or peak height) of analyte spiked into an actual blank sample (blank plasma for example) compared to being spiked into pure solvent, then analyzed by Q1 SIM. If the analyte concentration in the complex sample is known (e.g., a drug formulation), pure analyte can be added to pure solvent to the same concentration. However, if the analyte concentration in the complex sample is unknown and/or an analyte-free complex sample is not available, another way to estimate the matrix effect is to compare an isotope labeled internal standard spiked into the actual sample compared to being spiked into pure solvent. The assumption is made that the ionization efficiency and background are similar for both the analyte and internal standard. If the ratio of these two S/Ns is close to 1 , the matrix effect for the assayed analyte is minimal and indicates that the LC part of LC/MS method is optimized. In other words the maximal theoretical $\mathrm{S} / \mathrm{N}$ for this particular instrumental configuration has been achieved. (In rare circumstances, the matrix effect can increase signal [3, 7] and/or decrease background. As a result, the suppression coefficient can exceed the theoretical maximum of 1.0 ). This value, which we have termed the suppression coefficient, actually depends upon two factors: initial sample matrix complexity and chromatographic separation efficiency. However, as discussed above, these parameters usually can not be measured separately.

Defining the matrix suppression coefficient relative to the measured sensitivity of tandem mass spectrometry involves additional considerations. It is misleading to compare signal ratios obtained from analyte spiked into a matrix actual sample blank to pure solvent and measured by Q3 MRM (SRM) mode. This value is actually composed of the superposition of two factors: (1) efficiency of matrix effect reduction by LC purifica- tion and (2) MS/MS enhancement. These two parameters should be evaluated separately.

Evaluation of the degree of $\mathrm{S} / \mathrm{N}$ enhancement of an MS/MS method requires separate Q3 MRM (SRM) and (its precursor) Q1 SIM tests of the same sample in addition to measurements described above of the $\mathrm{S} / \mathrm{N}$ ratios of pure standard in solvent compared to standard in a complex matrix sample at Q1 SIM mode. The ratio between $\mathrm{S} / \mathrm{N}$ for the same complex sample measured at Q3 MRM (SRM) and (precursor) Q1 SIM tests is a criterion of overall MS/MS enhancement. We have termed this the tandem enhancement factor. If this number is low, perhaps 2 to 5 , it is possible to evaluate the likely success of transferring an MS/MS method to a single quadrupole instrument (if fragmentation of analyte is not mandatory) without significant loss in sensitivity. Based upon our experience, improvements in sample purification, by minimizing matrix effects, maximizes the inherent sensitivity of any and all mass spectrometers, including those relying on MS/MS for isolation of analytes from contaminants in the sample.

Although numerous papers in the LC/MS literature discuss matrix effects [3-12], we believe that assessing its importance during initial assay development has not been sufficiently emphasized in the research community. A current search of the LC/MS literature suggests that in manuscripts describing quantitative analysis from plasma, matrix effects are mentioned in only $20 \%$ of them, and are the subject of more in depth focus (appearing in the title, abstract, or keyword) in less than $5 \%$. Matrix effect usually causes signal suppression. It is a common LC/MS problem and, therefore, should be evaluated with each LC/MS method. Evaluation of matrix effects and chromatography/mass spectrometry efficiency is critical information about the quality of LC/MS method development; it is essential in the further optimization and ability to successfully transfer successfully methods across platforms to other mass spectrometers.

We would like to suggest that estimation of the strength of matrix effects (and the tandem enhancement factor) should be a mandatory part of LC/MS method development. We consider the suppression coefficient and enhancement factor as two parameters inherent to each LC/MS method; they are "quality control" variables of method optimization. Knowledge of the suppression coefficient and enhancement factor will facilitate subsequent assay validation.

\section{Acknowledgments}

The authors thank Dr. Kevin J. McHale and Dr. Justin Withers for helpful discussions and manuscript review. This work was supported by grants from the National Institutes of Health, 1 R01 DK61644-01, MO1-RR12248, and the American Diabetes Association. 


\section{References}

1. Taverniers, I.; De Loose, M.; Van Bockstaele, E. Trends in quality in the analytical laboratory. II. Analytical method validation and quality assurance. Trends Anal. Chem. 2004, 23(8), 535-552.

2. Zheng, J. J.; Lynch E. D.; Unger, S. E. Comparison of SPE and fast LC to eliminate mass spectrometric matrix effects from microsomal incubation products. J. Pharm. Biomed. Anal. 2002, 28, 279-285.

3. Stüber, M.; Reemtsma, T. Evaluation of three calibration methods to compensate matrix effects in environmental analysis with LC-ESI-MS. Anal. Bioanal. Chem. 2004, 378, 910-916.

4. Taylor, P. J. Matrix effects: The Achilles heel of quantitative high-performance liquid chromatography-electrospray-tandem mass spectrometry. Clin. Biochem. 2005, 38(4), 328-334.

5. Basilicata, P.; Miraglia, N.; Pieri, M.; Acampora, A.; Soleo, L.; Sannolo, N. Application of the standard addition approach for the quantification of urinary benzene. J. Chromatogr. B 2005, 818(2), 293-299.

6. Kloepfer, A.; Quintana, J.; B.; Reemtsma, T. Operational options to reduce matrix effects in liquid chromatographyelectrospray ionization-mass spectrometry analysis of aqueous environmental samples. J. Chromatogr. A 2005, 1067, 153160.
7. Zrostlikova, J.; Hajslova, J.; Poustka, J.; Begany, P. Alternative calibration approaches to compensate the effect of coextracted matrix components in liquid chromatography-electrospray ionization tandem mass spectrometry analysis of pesticide residues in plant materials. J. Chromatogr. A 2002, 973, 13-26.

8. Souverain, S.; Rudaz, S.; Veuthey, J.-L. Matrix effect in LCESI-MS and LC-APCI-MS with off-line and on-line extraction procedures. J. Chromatogr. A 2004, 1058, 61-66.

9. Alder, L.; Lüderitz, S.; Lindtner, K.; Stan, H.-J. The ECHO technique - the more effective way of data evaluation in liquid chromatography-tandem mass spectrometry analysis. J. Chromatogr. A 2004, 1058(1/2), 67-79.

10. Dams, R.; Huestis, M.; Lambert, W.; Murphy, C. Matrix effect in bioanalysis of illicit drugs with LC-MS/MS: Influence of ionization type, sample preparation, and biofluid. J. Am. Soc. Mass Spectrom. 2003, 14(11), 1290-1294.

11. Bonfiglio, R.; King, R.; Olah, T.; Merkle, K. The effects of sample preparation methods on variability of the electrospray ionization response for model drug compounds. Rapid Commun. Mass Spectrom. 1999, 13, 1175-1185.

12. Avery, M. Quantitative characterization of differential ion suppression on liquid chromatography/atmospheric pressure ionization mass spectrometric bioanalytical methods. Rapid. Commun. Mass Spectrom. 2003, 17, 197-201. 